\title{
The utility of mean platelet volume delta $(\triangle V P M)$ and mean platelet volume / platelet index as predictors of mortality in patients with Sepsis and Septic Shock.
}

\author{
DOI: $10.46981 /$ sjhnv2n2-011
}

Received in: April 1st, 2021

Accepted in: May 31th, 2021

\section{Serrano González America}

Department of Internal Medicine. Regional General Hospital No. 20, Tijuana, Baja California.

\section{Bautista López Federico del Jesús}

Department of Internal Medicine. Regional General Hospital No. 20, Tijuana, Baja California.

\section{Jimenez Ríos Eusebio}

Department of Internal Medicine. Regional General Hospital No. 1, Mérida, Yucatán.

\begin{abstract}
Background. Sepsis and septic shock are the most severe clinical expressions of infection, despite having characterized several decades ago, remain as a diagnostic challenging and this can delay treatment and increasing hospital cost secondary to this pathology.

Our objective was to determine the usefulness of the mean platelet volume delta ( $\triangle \mathrm{VPM})$ as a predictor of mortality.

Methodology. We performed an observational and descriptive study with 83 patients with Sepsis and / or septic shock criteria, from May 1, 2017 to August 1, 2017. Survival analysis was performed using Kaplan and Meier tables and was corroborated by the Long Rank test. The prognostic value through the proportional risk of Cox.

Results. Thirty-seven patients (44.6\%) died because of the septic process. A univariate Cox regression analysis revealed a MPV / platelet index at admission of less than 4 was associated with a significant reduction in the risk of mortality from sepsis or septic shock at 60 days (HR, 0.236; 95\% CI, 0.083 $0.671 ; \mathrm{p}=0.007)$. The DVPM was not significantly associated with a reduction in the risk of mortality (HR, 0.373, 95\% CI, 0.132-1.057, $\mathrm{p}=0.640$ ).

Conclusion. The platelet parameters are economical and available, providing diagnostic and prognostic results, avoiding costs, time and special tests. The VPM / PLQ index could be an effective "Alert System" in early sepsis.
\end{abstract}

Key words: Sepsis, septic shock, mean platelet volume, medium platelet volume delta.

\section{BACKGROUND}

Sepsis is a syndrome composed of physiological, pathological and biochemical alterations caused by an infection. In recent decades, it has been considered a public health concern that generates high costs, although the exact incidence is unknown, there are indicators that place this pathology as the main cause of mortality in critically ill patients worldwide ${ }^{2}$. Since the implementation of various protocols such as early goal-directed therapy, a decrease in mortality from sepsis has been achieved, however the 
risk of mortality and morbidity remains high. Which we can associate with a delay in diagnosis and treatment $^{3-4-5}$.

It is not uncommon for the hematological system to be affected by the significant inflammation present in critically ill patients. Hematological profiles including hematocrit, leukocyte count and platelet count have been used in widely accepted prognostic scales such as the sequenced organ failure assessment (SOFA) and the multiple organ dysfunction scale (MODS) ${ }^{6}$. Within the pathophysiology we find the release of pro and anti-inflammatory cytokines generating alterations in the coagulation cascade, such as prolongation of PT (prothrombin time) and aPTT (activated thromboplastin time), in addition to a decrease in platelets ${ }^{7-8}$. It is important to add that there is also peripheral non-immune destruction and marrow suppression which contribute to thrombocytopenia in septic patients ${ }^{9}$. The marked decrease in platelets correlates with the prognosis ${ }^{10}$. Therefore, several investigations have been interested in platelet indices, such as mean platelet volume (MPV). The mean platelet volume is a platelet index available since 1970, it is an accurate measure of platelet size and platelet reactivity ${ }^{11-12}$. Platelets with high MPV contain a greater number of granules and prothrombotic material, which conditions greater platelet aggregation and adhesion ${ }^{13}$. High MPV values usually reflect compensatory bone marrow production after stress-induced destruction in sepsis ${ }^{14}$. Numerous studies suggest what changes in MPV are observed during the infectious process, sepsis, coronary artery disease, cerebrovascular disease, venous and arterial thrombosis, and chronic inflammatory diseases ${ }^{15-16}$.

The use of serum biomarkers has the potential to significantly improve the clinician's ability to make a diagnosis, stratify risk, and provide optimal treatment for patients with sepsis. Recent findings suggest that mean platelet volume, $\triangle \mathrm{VPM}$, and MPV / Platelet ratio, may be useful as a prognostic biomarker in patients with sepsis. Ho Oh et al observed a superiority of the MPV / platelet index versus MPV alone in predicting mortality at 28 days ${ }^{17}$. Likewise, Zampieri found that non-surviving patients showed higher $\triangle$ VPM levels ${ }^{18}$.

Sepsis and its clinical spectra still continue to be one of the pathologies that represents the greatest complexity for clinicians. The delay in diagnosis and treatment conditions an increase in hospital stay days, a greater risk of morbidity and mortality, and an increase in the financial burden for the hospital. The agency for research and quality of health care lists sepsis as the most expensive condition treated in hospitals in the United States, establishing a cost of more than 20 billion in 2011, with annual increases of $11.9 \%$. This pathology continues to be the primary cause of death associated with infection despite advances in medicine. In countries like Mexico, sepsis represents 60-80\% of death per year.

Early detection and timely treatment of sepsis have an enormous impact on the prognosis of patients, which is why numerous investigations have tried to establish one or more biomarkers that can improve the outcome of sepsis. 
For this reason, it was considered in our study that the use of these platelet indices can be used as predictors of mortality in patients with sepsis and septic shock. In this way, we provide a reliable, accessible tool to carry out timely detection of patients with a higher risk of complications, with the aim of carrying out timely interventions that increase their probability of survival. It is important to emphasize that the availability of biomarkers that support the stratification of patients with sepsis is scarce in our hospital environment and not always accessible, which is why it is considered of vital importance to establish the reliability of tools such as $\triangle \mathrm{VPM}$ and MPV / platelet index, which are constantly available to the clinician 24 hours a day without the need for special blood sampling. Establishing these types of tools in the management of sepsis represents a significant cost containment, when compared with the costs produced by the use of other biomarkers such as lactate and procacyltonin.

The objective of this study was to determine the usefulness of the Mean Platelet Volume delta $(\triangle \mathrm{VPM})$ and the MPV / Platelet index as predictors of mortality in patients with Sepsis and Shock.

\section{PATIENTS AND STUDY DESIGN.}

An observational, longitudinal, prospective and descriptive study was carried out, with data obtained from patients with a diagnosis of Sepsis and septic shock, according to the third definition of Sepsis, admitted to the Regional General Hospital No. 1 "Lic. Ignacio García Téllez”. This study was reviewed and approved by the Local Research and Ethics Committee in Health Research, Hospital General Regional Numero 1, Yucatán.

Eighty-three patients older than 18 years admitted to the emergency department, internal medicine and intensive care in the period from May 1 to August 1, 2017, with a non-probabilistic sampling technique were included. A blood sample was taken from all patients for processing of hematic biometry and biochemical parameters at hospital admission and 72 hours. Thus, information was also collected from the clinical record, laboratory results, measured hemodynamic parameters, and the SOFA and APACHE II scale score was calculated. With the results of MPV and platelets at admission and at 72 hours, the $\triangle$ VPM and MPV / platelet index were calculated. Sepsis was defined as life-threatening organ dysfunction, caused by an alteration in the host's response to infection, always emphasizing a nonhomeostatic response to infection. Septic shock was defined as a subset of sepsis in which underlying abnormalities are found in circulation and cellular metabolism, which can be objectively characterized as persistent hypotension with mean arterial pressure values $<65 \mathrm{mmHg}$ and / or hyperlactatemia $>2$ mmol a despite the use of vasopressors and adequate fluid resuscitation. The $\triangle$ VPM and MPV / Platelet index were defined as the percentage of change in MPV in the first 72 hours after admission $(\triangle \mathrm{MPV}=$ ([72 hrs MPV - admission MPV] / admission MPV) x 100) and MPV / Platelet index as [VPM / Platelet (count / 1000)] x 100, respectively. 


\section{STATISTIC ANALYSIS}

In the statistical analysis, it was performed in means, modes, medians, minimum and maximum ranges, as well as standard deviations for continuous variables, while categorical variables were expressed with numbers and percentages. Mortality was assessed as the primary outcome. The comparison of the parameters obtained between the groups of survivors and non-survivors was performed using the Mann Whitney $U$ test for continuous variables and chi square for categorical variables. The correlation between $\triangle \mathrm{VPM}, \mathrm{MPV}, \mathrm{MPV} /$ Platelet index at admission, APACHE II score and SOFA were processed using a Spearman correlation analysis. Survival was analyzed using Kaplan and Meyer graphs and corroborated using the Long Rank test. The prognostic value of $\triangle \mathrm{VPM}$ and MPV / Platelet index at admission and 72 hours was evaluated using the Cox proportional hazard expressed in Hazard Ratios (HR) with a confidence interval of $95 \%$. The value of $\mathrm{P}<0.05$ was considered statistically significant. Statistical analysis was performed with SPSS for Windows version 22.0 (IBM corporation).

\section{RESULTS}

The mean age of the patients studied was 64.89 years ( +13.93 years). $50.6 \%$ (42 patients) were female and $49.4 \%$ (41 patients) were male. Of the total of patients recruited, 55.4\% (46 patients) were censored; on the contrary, 44.6\% (37 patients) died as a direct cause of the septic process. The main etiology of the septic process was pulmonary infection with $28.9 \%$ (24 cases), followed by urinary tract infections with $26.5 \%$ (22 cases), abdominal infections with $25.3 \%$ (21 cases) and finally soft tissue infections with $19.3 \%$ (16 cases).

The mean MPV / platelet index at admission was 7.5 and 12.7 at 72 hours in the group of nonsurvivors and in the survivors it was 4.6 and 5.5 respectively (p 0.001 and $p<0.001$ ). Regarding the $\triangle \mathrm{VPM}$, it was $8.0 \mathrm{fL}$ in the survivors, increasing considerably to $8.6 \mathrm{fL}$ in the non-survivors ( $\mathrm{p} 0.515$ ) Table 1.

The Kaplan and Meier graphs described the probability of survival at 60 days, finding greater survival in patients with $\triangle \mathrm{VPM}<3 \mathrm{fL}$ (p 0.049). Patients with an MPV / platelet index at admission and

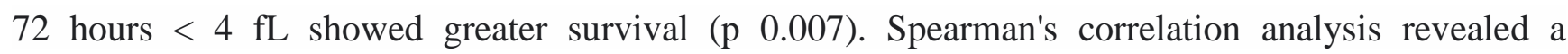
significantly positive correlation between the MPV / platelet index at admission and at 72 hours $(\mathrm{r}=$ 0.719; $\mathrm{p}=<0.001)$. Likewise, the MPV / platelets index at 72 hours showed a significantly positive correlation with the SOFA score at admission $(r=0.320 ; p=0.004)$. In contrast, the $\triangle$ VPM did not have a significant correlation with any of the variables. A univariate Cox regression analysis revealed that an MPV / platelet index on admission $<4$ was associated with a significant reduction in the risk of mortality from sepsis or septic shock at 60 days (HR, 0.236; 95\% CI, 0.083-0.671; $\mathrm{p}=0.007)$. In addition, the 
MPV / platelet index at 72 hours < 4 was also associated with a significant reduction in the risk of mortality from sepsis or septic shock at 60 days (HR, 0.190; 95\% CI, 0.058-0.625; $\mathrm{p}=0.006) . \Delta \mathrm{VPM}$ was not significantly associated with a reduction in the risk of mortality from sepsis or septic shock at 60 days (HR, 0.373; 95\% CI, 0.132-1.057; $\mathrm{p}=0.640)$. In a multivariate analysis, the MPV / platelet index on admission revealed to be a significant risk factor for mortality from sepsis or septic shock when adjusted for age, sex, APACHE II score, SOFA score, degree of acute kidney injury, and origin. of the infectious site (HR, 2.919; 95\% CI, 1.254-6.797; $\mathrm{p}=0.013)$. Additionally, the MPV / platelets index at 72 hours was similarly shown to be a significant risk factor when adjusted with the same variables (HR, 7.289; 95\% CI, 1.784-29.786; $\mathrm{p}=0.006)$.

\section{DISCUSSION}

Due to the impact of septic processes, it is necessary to establish accessible prognostic markers to discriminate severity in patients with sepsis and septic shock. There is ample literature in which an attempt has been made to document the relevance of platelet parameters in patients diagnosed with sepsis. Among the parameters studied are MPV, platelet index, platelet distribution width, delta MPV, MPV / Platelet index, among others. This study was carried out with the intention of establishing the correlation between MPV / Platelet index and MPV delta with mortality in patients diagnosed with sepsis and septic shock. Venkata et al. Found an association between thrombocytopenia and mortality, as well as a higher incidence of acute renal failure in contrast to patients who do not present thrombocytopenia; however, despite this, they did not find an association with the isolated platelet count at admission as a prognostic factor in patients with sepsis ${ }^{19}$. The platelet count in our population of survivors and nonsurvivors did have a significant difference both at admission and at 72 hours. Regarding MPV values at admission and at 72 hours, we also found a significant difference between both groups. On the other hand, the MPV delta did not show a significant difference between both groups, although it showed a relationship with mortality when figures greater than $3 \mathrm{fL}$ were presented, the risk ratio was not significant. In the same way, the MPV / Platelet index at admission and 72 hours showed an association with an increase in mortality when figures greater than $4 \mathrm{fL}$ were obtained, with a significant risk ratio for both the index obtained at admission and at 72 hours.

In contrast to our results, Kim et al demonstrated that both the platelet count and the MPV value in non-surviving patients with sepsis and septic shock were higher than in the survivors during the first 72 hours $^{20}$. Now the increase in baseline MPV levels had tried to establish it as an independent risk factor for mortality, seeking to establish this. Oh et al found that both MPV and platelet count alone do not predict 28-day mortality in patients with sepsis ${ }^{17}$. Although the follow-up in our study was not as long, higher levels were evidenced in non-survivors, however it was not possible to establish a 
prognostic factor for mortality. Kim et al found that MPV delta was a significantly useful marker for predicting 28-day mortality ${ }^{20}$. Contrary to what was observed in our study, where no utility was shown to predict mortality from the MPV delta.

Within the literature, there is less evidence regarding the MPV / Platelet index, Azab et al were the first to establish superiority of the MPV / Platelet Index over MPV alone, as a prognostic factor in patients with AMI without ST elevation ${ }^{21}$. Once this relationship with the level of inflammation present in other pathologies has been established, in our study, a univariate analysis revealed that an MPV / Platelet index at admission and 72 hours $<4 \mathrm{fL}$ are useful as predictors of survival at 60 days. On the contrary, when performing a multivariate analysis adjusted to different confounders, it was found that MPV / Platelet index > $4 \mathrm{fL}$ both at admission and 72 hours were related to an increase in mortality at 60 days in patients with sepsis and septic shock. Similarly, a high correlation was observed between the MPV / Platelets index at admission and 72 hours with the SOFA scale, MPV levels and platelet counts.

This study has several limitations, firstly, the recruited population was from a single hospital, and secondly, there were variants that were not taken into consideration, such as the smoking habit of the patients, the intake of drugs such as antiplatelet agents and non-steroidal anti-inflammatory drugs 710 days prior to admission, since these drugs can modify MPV values.

\section{CONCLUSIONS}

In contrast to other $\triangle$ VPM studies, our study showed that it is not useful for predicting mortality, however, in patients with sepsis and septic shock an MPV / Platelet index greater than $4 \mathrm{fL}$ did prove useful as a predictor of mortality both when admission as at 72 hours, indicating the potential need for more intensive monitoring and management due to its close association with early mortality.

The platelet count as well as its indices are inexpensive and are available in routine laboratories, so serial measurements could be performed in patients with sepsis and septic shock, providing results for both diagnosis and severity, avoiding costs, long times and special tests. additionally, for all this the MPV / Platelet index could be an effective "Alert System" in early stages of sepsis.

A greater number of studies are required in the future to validate the usefulness of platelet indices as prognostic markers in patients with sepsis, in addition to establishing reference values for MPV, as well as the association of these values with the etiological agent of infectious processes. 


\section{REFERENCES}

1. Mervyn Singer, MD, FRCP; Clifford S. Deutschman, MD, MS; ChristopherWarren Seymour et al. The Third International Consensus Definitions for Sepsis and Septic Shock (Sepsis-3). JAMA. 2016; 315 (8): 801-810.

2. Vincent J-L, Marshall JC, Namendys-Silva SA, et al; ICON Investigators. Assessment of the worldwide burden of critical illness: the Intensive Care Over Nations (ICON) audit. Lancet RespirMed. 2014; 2 (5): 380-386

3. Rivers E, Nguyen B, Havstad S, Ressler J, Muzzin A, Knoblich B, Peterson E, Tomlanovich M: Early goal-directed therapy in the treatment of severe sepsis and septic shock. N Engl J Med 345: 1368-1377, 2001

4.Peake SL, Delaney A, Bailey M, Bellomo R, Cameron PA, Cooper DJ, Higgins AM, Holdgate A, Howe BD, Webb SA, et al .: Goal-directed resuscitation for patients with early septic shock. N Engl J Med 371: 1496-1506, 2014.

5. Yealy DM, Kellum JA, Huang DT, Barnato AE, Weissfeld LA, Pike F, Terndrup T, Wang HE, Hou PC, LoVecchio F, et al .: A randomized trial of protocol-based care for early septic shock. N Engl J Med 370: 1683-1693, 2014.

6. J.-L. Vincent, R. Moreno, J. Takala et al., "The SOFA (Sepsis-Related Organ Failure Assessment) score to describe or dysfunction / failure. On behalf of the Working Group on Sepsis- Related Problems of the European Society of Intensive Care Medicine, 'Intensive Care Medicine, vol. 22, no. 7, pp. 707$710,1996$.

7. Becchi C, Al Malyan M, Fabbri LP, Marsili M, Boddi V, Boncinelli S. Mean platelet volume trend in sepsis: is it a useful parameter? Minerva Anesthesiol. 2006; 72 (9): 749-56.

8. Gao Y, Li Y, Yu X, Guo S, Ji X, et al. (2014) The Impact of Various Platelet Indices as Prognostic Markers of Septic Shock. PLoS ONE 9 (8): e103761. doi: 10. 1371 / journal.pone.0103761.

9.Guclu E, Durmaz Y, Karabay O. Effect of severe sepsis on platelet count and their indices. African Health Sciences Vol 13 Issue 2 June 2013.

10. Zakynthinos SG, Papanikolaou S, Theodoridis T, Zakynthinos EG, Christopoulou-Kokkinou V, et al. (2004) Sepsis severity is the major determinant of circulating thrombopoietin levels in septic patients. Crit Care Med 32: 1004-10.

11. Hande Aydemir, Nihal Piskin, Deniz Akduman et al. Platelet and mean platelet volume kinetics in adult patients with sepsis. Platelets, 2012; Early Online: 1-6

12. Sansanayudh N, Anothaisintawee T, Muntham D, McEvoy M, Attia J, Thak-kinstian A: Mean platelet volume and coronary artery disease: a systematic review and meta-analysis. Int J Cardiol 175: 433-440, 2014.

13. C. B. Thompson, J. A. Jakubowski, P. G. Quinn, D. Deykin, and C. R. Valeri, "Platelet size as a determinant of platelet function," The Journal of Laboratory and Clinical Medicine, vol. 101, no. 2, pp. 205-213, 1983. 
14. Kim CH, Kim SJ, Lee MJ, Kwon YE, Kim YL, Park KS, Ryu HJ, Park JT, Han SH, Yoo TH, et al $\therefore$ An increase in mean platelet volume from baseline is associated with mortality in patients with severe sepsis or septic shock. PLoS One 10: e0119437, 2015.

15. Chu SG, Becker RC, Berger PB, Bhatt DL, Eikelboom JW, Konkle B, Mohler ER, Reilly MP, Berger JS: Mean platelet volume as a predictor of cardiovascular risk: a systematic review and meta-analysis. J Thromb Haemost 8: 148-156, 2010.

16. Azab B, Torbey E, Singh J, Akerman M, Khoueiry G, McGinn JT, Widmann WD, Lafferty J: Mean platelet volume / platelet count ratio as a predictor of long-term mortality after non-ST-elevation myocardial infarction. Platelets 22: 557-566, 2011.

17. Geun Ho Oh, Sung Phil Chung, Yoo Seok Park et al. Mean platelet volume to platelet coins ratio as a promising predictor of early mortality in severe sepsis. SHOCK VOL. 47, No. 3. 2016.

18. Fernando G Zampieri, Otavio T Ranzani, Viviane Sabatoski et al. An increase in mean platelet volume after admission is associated with higher mortality in critically ill patients. Zampieri et al. Annals of Intensive Care 2014, 4:20.

19. Ventaka C, Kashyap R, Farmer JC, Afessa B: Thrombocytopenia in adult patints with sepsis: incidence, risk factors and its association with clinical outcome. J Intensive Care 1: 9, 2013.

20.Kim CH, Kim SJ Lee MJ, Kwon YE, et al. : An increase in mean platelet volume from baseline is associated with mortality in patients with severe sepsis or septic shock. PLoS One 10: e0119437, 2015

21. Azab B, Torbey E, Singh J, et al .: Mean platelet volume / platelet count ratio as a predictor of lonfterm mortality after non-ST -elevation myocardial infarction. Platelets 22: 557-566,201. 
Table 1. Clinical characteristics and biochemical variables

\begin{tabular}{|c|c|c|c|c|}
\hline Variable & Total $(n=83)$ & $\begin{array}{l}\text { Survivors } \\
(n=46)\end{array}$ & $\begin{array}{l}\text { Non survivors } \\
\quad(\mathbf{n}=\mathbf{3 7})\end{array}$ & $\begin{array}{l}\text { Value of } \\
P\end{array}$ \\
\hline \multicolumn{5}{|l|}{ Demographic data } \\
\hline Age & $64.89 \pm 13.93$ & $64.22 \pm 13.92$ & $65.46 \pm 13.67$ & 0.851 \\
\hline APACHE II & $17.21 \pm 6.24$ & $16.43 \pm 6.62$ & $17.82 \pm 5.48$ & 0.258 \\
\hline SOFA & $8.06 \pm 2.52$ & $7.51 \pm 2.60$ & $8.63 \pm 2.36$ & 0.007 \\
\hline \multicolumn{5}{|l|}{ Hematic cytometry data } \\
\hline Leukocytes ( x 103/ mm ${ }^{3}$ ) & $19.23 \pm 7.66$ & $17.98 \pm 7.46$ & $19.79 \pm 7.84$ & 0.313 \\
\hline Hemoglobin ( g/dL) & $10.24 \pm 2.27$ & $10.21 \pm 2.35$ & $10.58+2.36$ & 0.531 \\
\hline $\begin{array}{l}\text { Platelets admission }\left(\times 10^{3} /\right. \\
\left.\mathrm{mm}^{3}\right)\end{array}$ & $\begin{array}{c}218.71 \pm \\
117.20\end{array}$ & $\begin{array}{l}254.78 \pm \\
118.08\end{array}$ & $174.13 \pm 103.44$ & 0.002 \\
\hline Platelets 72 hrs $\left(\mathrm{x} 10^{3} / \mathrm{mm}^{3}\right)$ & $\begin{array}{c}173.48 \pm \\
107.80\end{array}$ & $\begin{array}{l}222.92 \pm \\
115.96\end{array}$ & $122.23 \pm 67.35$ & $<0.001$ \\
\hline MPV admission (fL) & $8.88 \pm 0.84$ & $8.63 \pm 0.85$ & $9.18 \pm 0.79$ & 0.014 \\
\hline MPV 72 hrs (fL) & $9.03 \pm 1.03$ & $8.49 \pm 0.94$ & $9.66 \pm 0.75$ & $<0.001$ \\
\hline$\Delta \mathrm{MPV}(\mathrm{fL})$ & $8.63 \pm 6.34$ & $8.09 \pm 7.01$ & $8.67 \pm 5.16$ & 0.515 \\
\hline MPV/Platelets admission & $5.85 \pm 5.08$ & $4.64 \pm 3.78$ & $7.58 \pm 6.24$ & 0.001 \\
\hline MPV/Platelets 72 hrs. & $8.98 \pm 11.14$ & $5.5 \pm 4.89$ & $12.78 \pm 14.6$ & $<0.001$ \\
\hline \multicolumn{5}{|c|}{$\begin{array}{l}\text { Data are means and standard deviations. APACHE II Acute Physiology and Chronic } \\
\text { Health Evaluation II; SOFA Sequential Organ Failure assessment; Mean Platelet Volume } \\
\text { MPV. } \\
\text { The value of P compares between survivors and non-survivors. }\end{array}$} \\
\hline
\end{tabular}


Figure 1. Kapla-Meier. Probability of survival at 60 days.

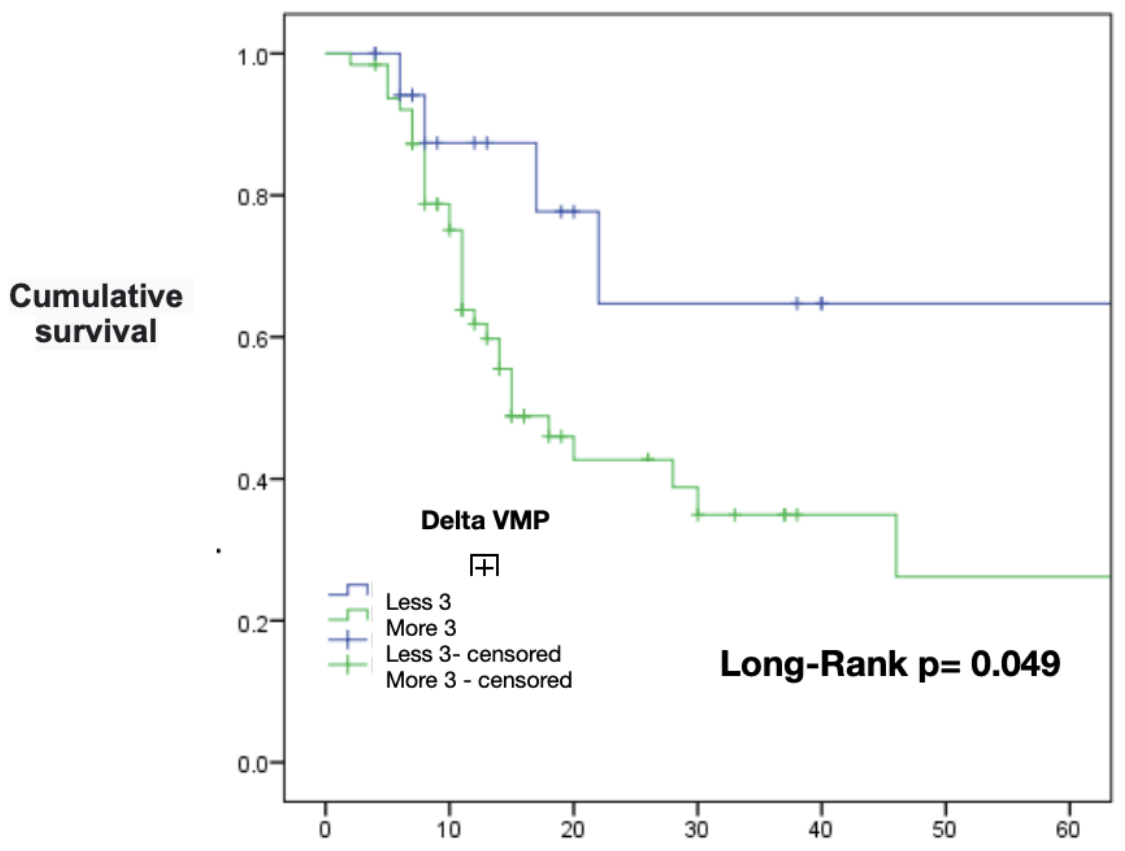

Survival in days.

Figure 2. Kapla-Meier. Probability of survival at 60 days.

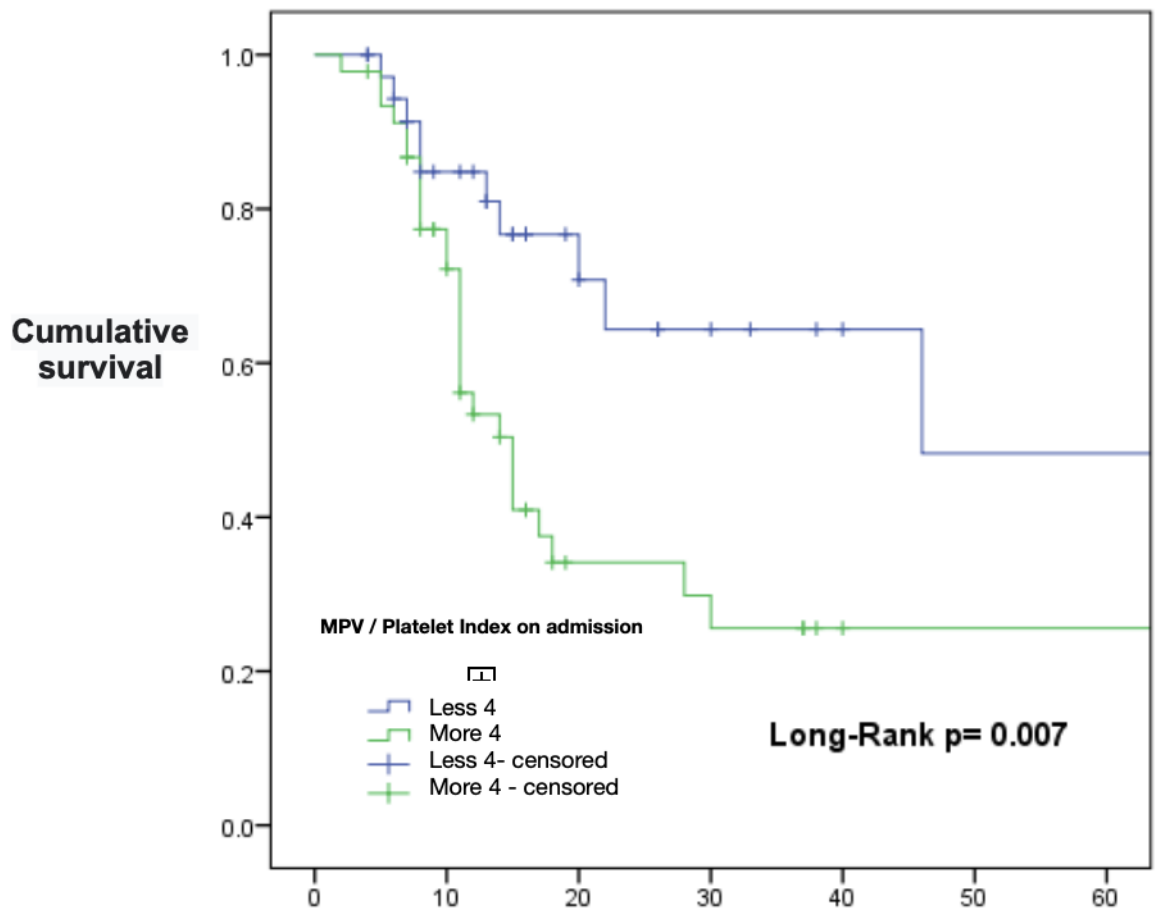

Survival in days. 
Figure 3. Kapla-Meier. Probability of survival at 60 days.

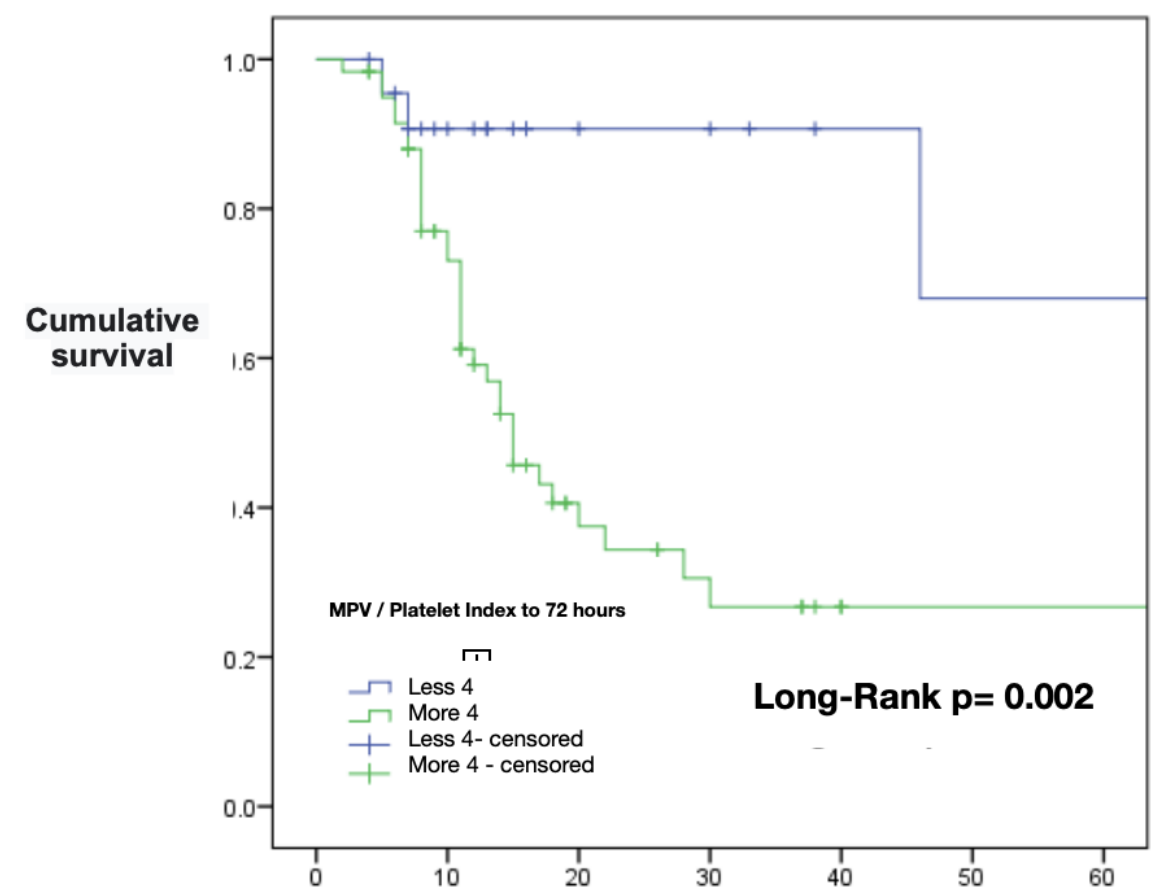

\section{Survival in days.}

Table 2. Correlation between $\triangle$ VPM, admission MPV/platelet index, MPV/ platelet $72 \mathrm{hrs}$ and variables.

\begin{tabular}{|c|c|c|c|c|c|c|}
\hline \multirow[t]{2}{*}{ Variable } & \multicolumn{2}{|c|}{ DVPM } & \multicolumn{2}{|c|}{$\begin{array}{c}\text { Admission } \\
\text { MPV/Platelet } \\
\text { index }\end{array}$} & \multicolumn{2}{|c|}{$\begin{array}{c}72 \text { hours } \\
\text { MPV/Platelet } \\
\text { index }\end{array}$} \\
\hline & $\mathbf{r}$ & $\begin{array}{l}\text { Valor } \\
\mathbf{P}\end{array}$ & $\mathbf{r}$ & $\begin{array}{c}\text { Valor } \\
\mathbf{p}\end{array}$ & $\mathbf{r}$ & $\begin{array}{c}\text { Valor } \\
\mathbf{p}\end{array}$ \\
\hline Age & -0.140 & 0.902 & -0.340 & 0.761 & -0.067 & 0.550 \\
\hline APACHE II & -0.930 & 0.402 & -0.009 & 0.935 & 0.080 & 0.489 \\
\hline SOFA & -0.770 & 0.500 & 0.080 & 0.482 & 0.320 & 0.004 \\
\hline Leukocytes $\left(\right.$ x $\left.10^{3} / \mathrm{mm}^{3}\right)$ & -0.120 & 0.285 & -0.021 & 0.854 & 0.048 & 0.669 \\
\hline Hemoglobin ( g/dL) & 0.350 & 0.752 & -0.047 & 0.675 & -0.016 & 0.888 \\
\hline $\begin{array}{l}\text { Platelets admission } \times 10^{3} \text { / } \\
\mathrm{mm}^{3}\end{array}$ & -0.079 & 0.479 & -0.988 & 0.000 & -0.711 & 0.000 \\
\hline $\begin{array}{l}\text { Platelets } 72 \mathrm{hrs}\left(\times 10^{3} /\right. \\
\left.\mathrm{mm}^{3}\right)\end{array}$ & -0.180 & 0.103 & -0.724 & 0.000 & -0.985 & 0.000 \\
\hline MPV admission (fL) & -0.147 & 0.185 & 0.338 & 0.002 & 0.235 & 0.032 \\
\hline MPV 72 hrs (fL) & 0.007 & 0.949 & 0.432 & 0.000 & 0.590 & 0.000 \\
\hline$\Delta \mathrm{MPV}(\mathrm{fL})$ & N/A & N/A & 0.053 & 0.633 & 0.161 & 0.147 \\
\hline MPV/Platelets admission & 0.053 & 0.633 & N/A & N/A & 0.719 & 0.000 \\
\hline
\end{tabular}




$\begin{array}{lllllll}\text { MPV/Platelets } 72 \text { hrs. } & 0.161 & 0.147 & 0.719 & 0.000 & \text { N/A } & \text { N/A }\end{array}$

APACHE II Acute Physiology and Chronic Health Evaluation II; SOFA Sequential Organ Failure assessment; MPV Mean Platelet Volumen. 